\title{
Research Paper: The Relationship Between Balance and Activities of Daily Living With the Quality of Life of Patients With Relapsing-Remitting Multiple Sclerosis
}

\author{
Mansour Noori ${ }^{1} \odot$, Seyed Ali Hosseini ${ }^{1}$, Vali Shiri $^{2,3},{ }^{*}$ Nazila Akbarfahimi ${ }^{1} \mathrm{C}$
}

1. Department of Occupational Therapy, University of Social Welfare and Rehabilitation Sciences, Tehran, Iran

2. Department of Occupational Therapy, School of Rehabilitation Sciences, Isfahan University of Medical Sciences, Isfahan, Iran.

3. Musculoskeletal Research Center, Isfahan University of Medical Sciences, Isfahan, Iran.

\begin{tabular}{|c|c|}
\hline $\begin{array}{l}\text { Use your device to scan } \\
\text { and read the article online }\end{array}$ & dteation Noori M, Hosseini SA, Shiri V, Akbarfahimi N. [The Relationship Between Balance and Activities of Daily Living \\
\hline 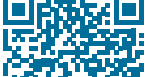 & $\begin{array}{l}\text { With the Quality of Life of Patients With Relapsing-Remitting Multiple Sclerosis (Persian)]. Archives of Rehabilitation. 2019; } \\
\text { 19(4):292-313. http://dx.doi.org/10.32598/rj.19.4.292 }\end{array}$ \\
\hline arsing & d tej/http://dx.doi.org/10.32598/rj.19.4.292 \\
\hline
\end{tabular}

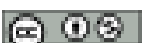

Received: 06 Aug 2018

Accepted: 28 Nov 2018

Available Online: 01 Jan 2019

\section{ABSTRACT}

Objective Multiple Sclerosis (MS) as a progressive disease has remarkable consequences on the patient's Quality of Life. According to the surveys, the Quality of Life in the people with MS is lower than the healthy people that is more prominent in physical, self-caring and daily performance activities. There are various reasons for this low Quality of Life such as lack of definitive treatment, relapsing-remitting periods, and unpredictable relapsing periods. Although, the effect of personal, social and economic factors on the Quality of Life has already been investigated, no study has been conducted in order to survey balance and activity of daily living on the Quality of Life in people with MS, Thus, the present study aims to investigate the relationship between balance and activity of daily living with the Quality of Life in this group of patients.

Materials \& Methods This investigation is a cross-sectional study that 40 patients with the relapsing- remitting MS were selected by convenience sampling method in Isfahan City, Iran. The written consent was taken from all patients and their families. The demographic characteristics of the patients were kept private and the patients could leave the investigation whenever they wanted. The inclusion criteria were as follows: the patients' willingness to participate in the study, verification of relapsing-remitting MS diagnosis, the patients referred to Al-Zahra and Kashani hospitals, age range 20-40 years, minimum of two attacks during the last 24 months, scored less than 505 in Expanded Disability Status Scale (EDSS), affection to MS moderate type in terms of disability diversity and the disease signs, able to cooperate with the study by means of MMSE test. The exclusion criteria were as follows: Unwillingness to continue the study, participating in the similar studies during 3 months ago which affected their Quality of Life, balance or activity of daily living, visual or auditory deficits, recurrence of the disease or worsening the disability from moderate to severe, any simultaneous disorder like seizure. The assessment tools were Expanded Disability Status Scale (EDSS), Berg Balance Scale, Barthel index, and Quality of Life Questionnaire SF-36. The collected data was analyzed by Pearson correlation and regression analyses in SPSS 20.

Results There is a positive and significant relationship $(P<0.01)$ between physical activity $(66 \%)$, physical role functioning (98\%), physical dimensional scale (93\%), and the Quality of Life. There wasn't any significant relationship between balance and other Quality of Life components. Self-care has a positive and significant relationship with physical activity (60\%), and physical dimensional scale (56\%) components. In addition, there is a positive and significant relationship between physi-

\section{* Corresponding Author:}

Nazila Akbarfahimi, PhD.

Address: Department of Occupational Therapy, University of Social Welfare and Rehabilitation Sciences, Tehran, Iran.

Tel: +98 (21) 22180063

E-Mail: fahimi1970@yahoo.com 
Keywords:

Multiple Sclerosis,

Balance, Quality of

Life, Activities of

daily living cal activity (75\%), physical role functioning (45\%) and physical dimensional scale (67\%). However, there was not any significant relationship between self-care and daily performance with the other Quality of Life components.

Conclusion The results indicate that the balance and independence in the activities of daily living can predict the Quality of Life in the patients with MS. Accordingly, in the interventions done for rehabilitation of MS patients, important factors such as Quality of Life and daily living skills should be taken into account. It seems that the vestibular system rehabilitation and the instruction of activity of daily living can improve the physical dimension in the MS patients' lives. 
This Page Intentionally Left Blank 


\title{
بررسى رابطه بين تعادل وفعاليتهاى روزمرهزندكى باكيفيت زندتى در افرادبامالتيبل اسكلروزيس عودفروكش بروسي
}

\author{
منصور نورى' •، سيد على حسينى'، ولى شيرى "،r. •نازيلا اكبر فهيمى'

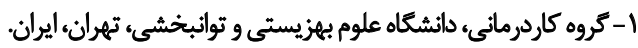

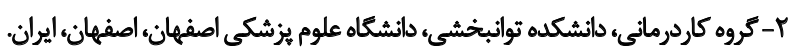

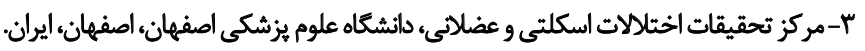

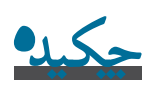

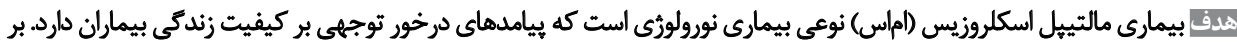

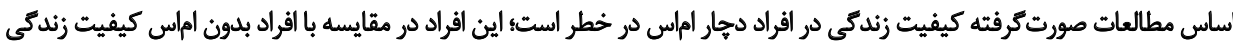

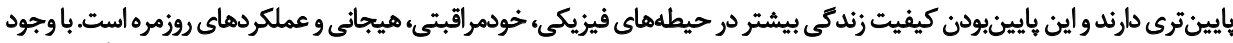

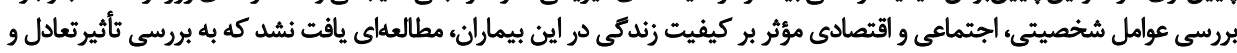

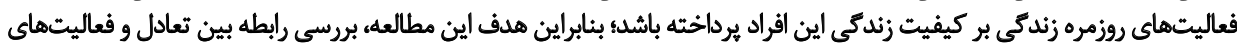

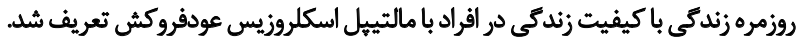

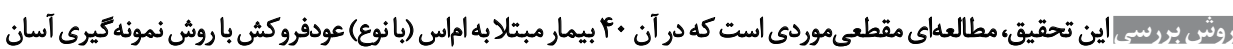

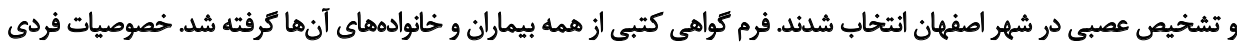

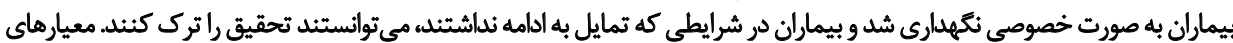

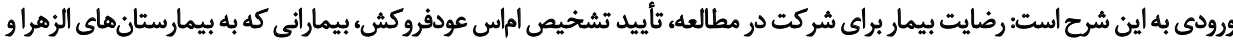

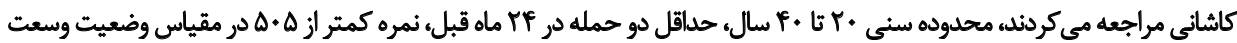

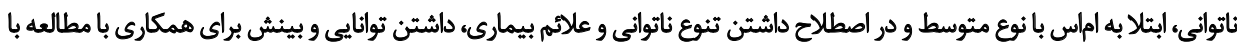

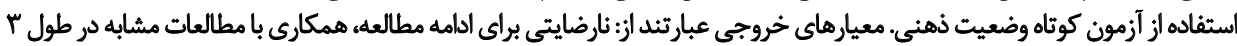

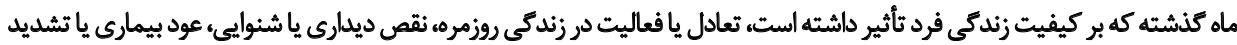

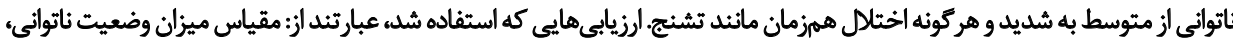

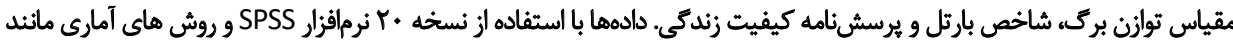

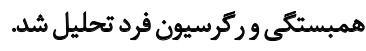

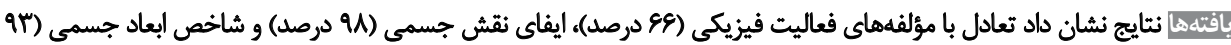

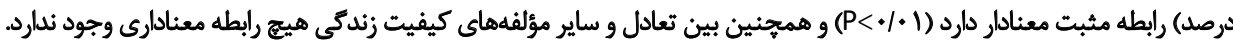

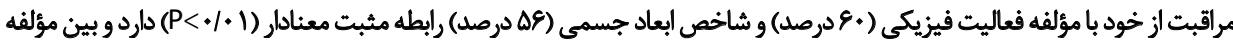

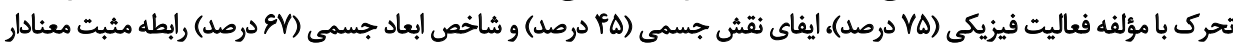

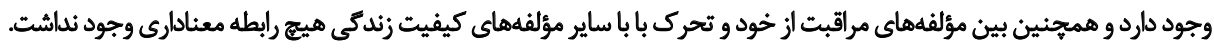

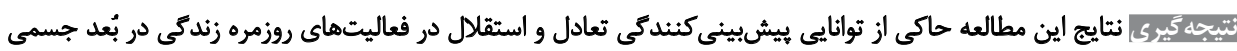

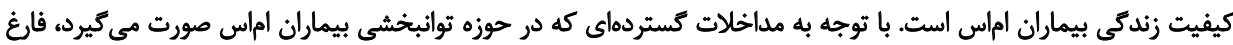

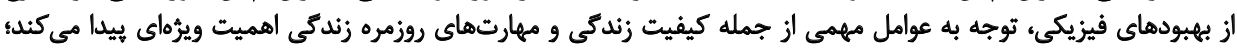

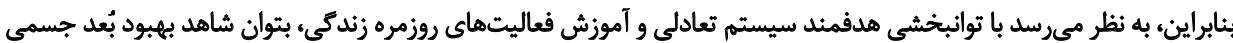

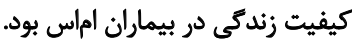

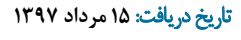

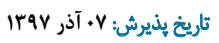

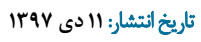

كليدوأوها: مالتييل اسكلروزيس،

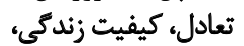
فعاليتهاى روزمره زندىى

도.

$$
\begin{aligned}
& \text { " نويسنده مسئُول: }
\end{aligned}
$$

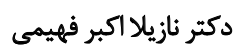

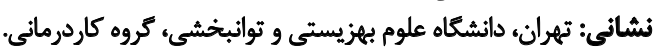

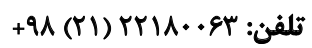

$$
\begin{aligned}
& \text { راياثامه: fahimi1970@yahoo.com }
\end{aligned}
$$




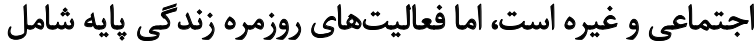

مقدمه

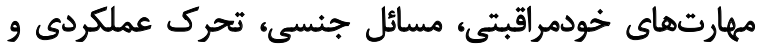

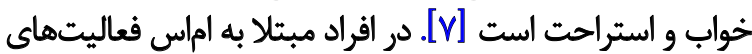

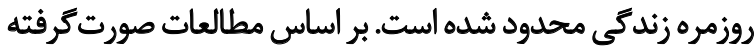

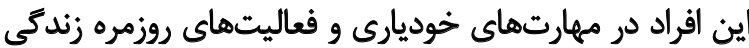

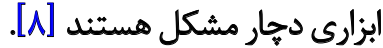
سنغلاجى و همكاران به بررسى تأثير تمرينات تركيبى، ازئ جمله

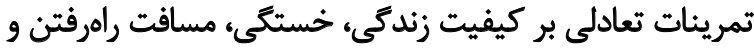

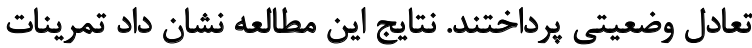

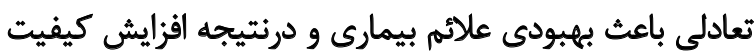

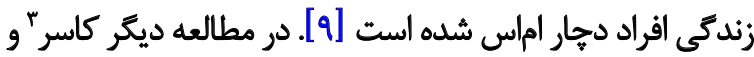

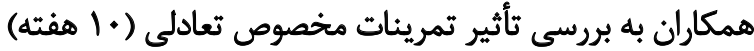

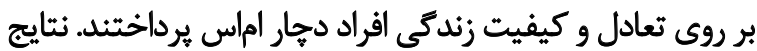

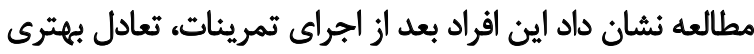

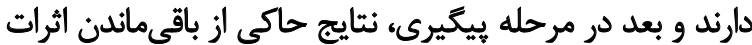

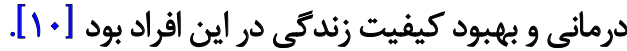
بر اساس مطالعه ميلينز" و همكاران كه به بررسى رابطه

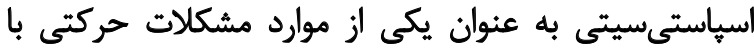

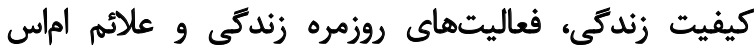

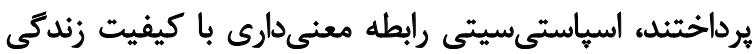

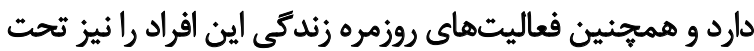

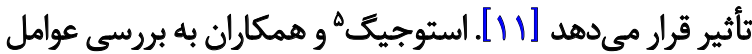

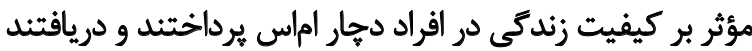

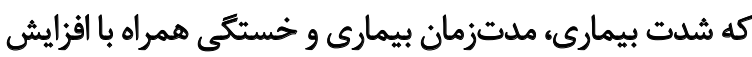
اضطراب و افسردكى باكيفيت زندكى اين افراد ارتباط دارد [ [ I ]

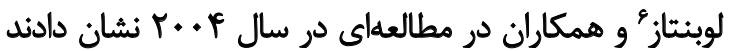

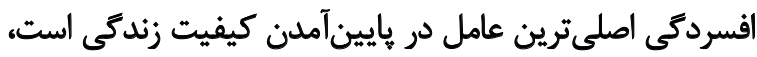

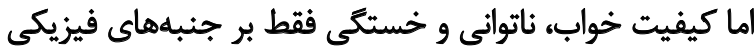

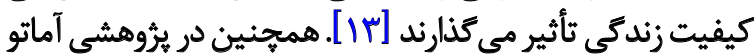

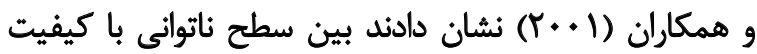

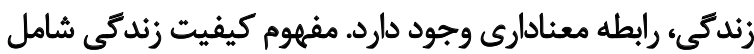

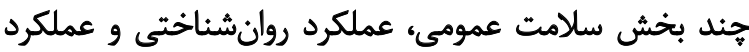

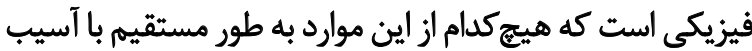

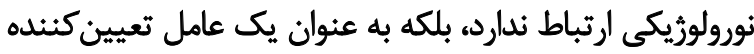

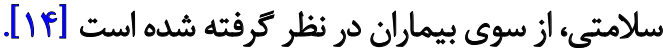

افراد با اماس كيفيت زندكى بإيينى دارند و اين پايينينودن

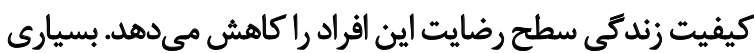

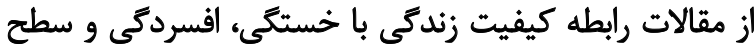

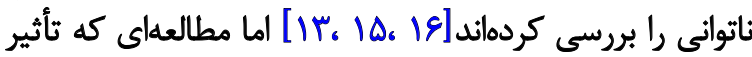

\footnotetext{
3. Kasser

4. Milinis

5. Ostojic

6. Lobentanz
}

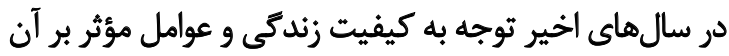

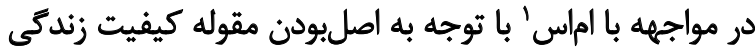

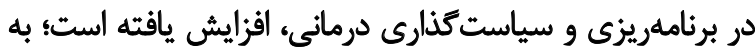

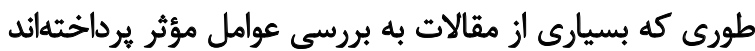

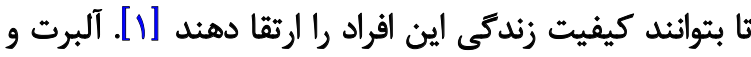

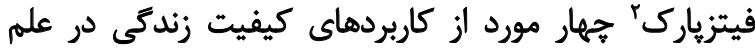

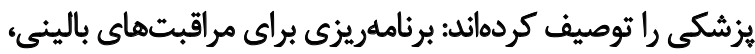

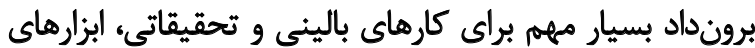

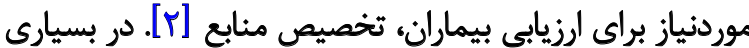

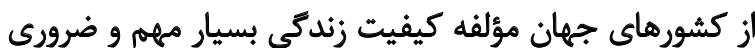

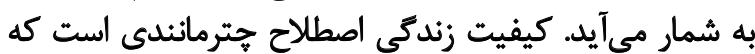

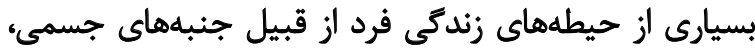

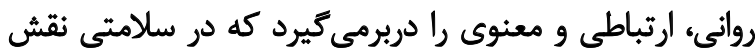

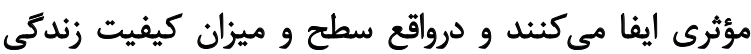

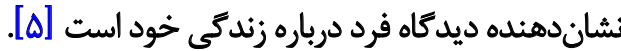

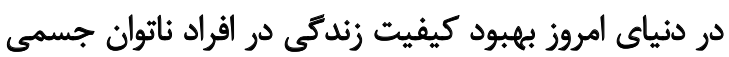

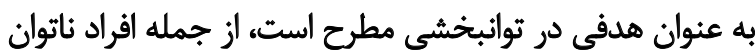

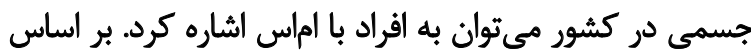

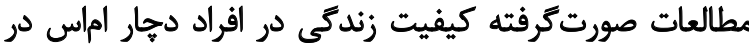

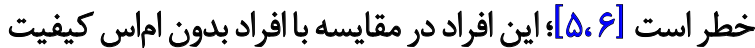

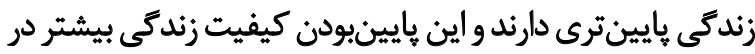

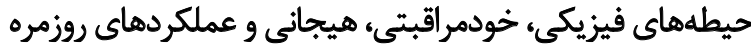

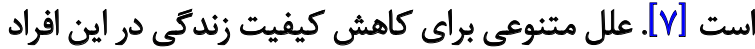

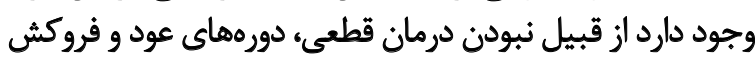
و مراحل عود بيشبينىنإيذير [هان].

اماس اختلالى نورولوريكيى است كه تأثيرات منفى روى فرد و

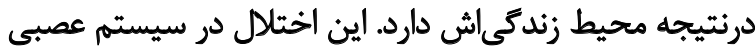

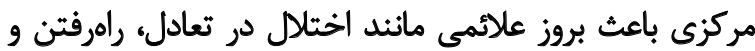

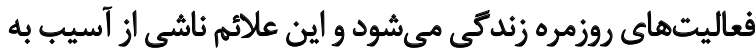

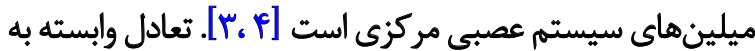

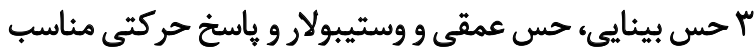

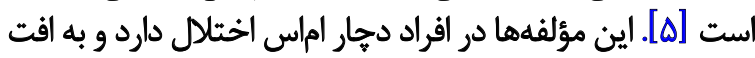

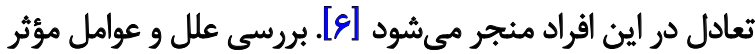

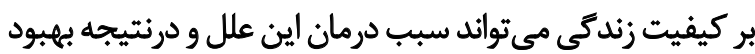
كيفيت زندگى در افراد دركير اماس شودي فعاليتهاى روزمره زندكى به فعاليتهايى هايه و فعاليتهاى

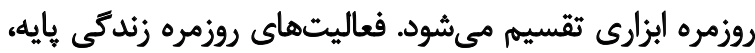

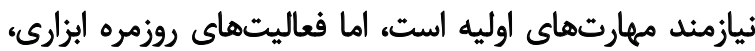

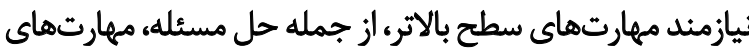


است كه اجازه بررسى وضعيت ناتوانى بيمار را به ما مي دهد. در

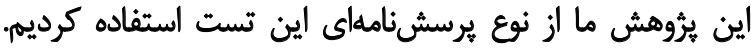

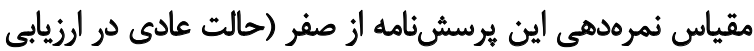

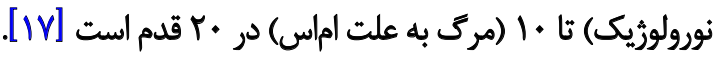

\section{يرسشنامه فعاليتهاى روزمره زندكى بارثل}

شاخص بارتل براي ارزيابى فعاليتهاى روزمره زندكى است

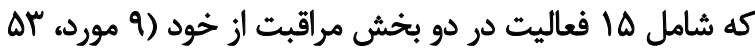

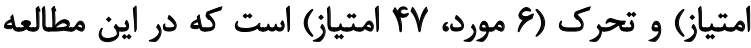

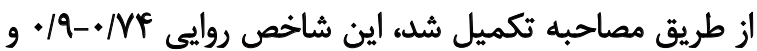

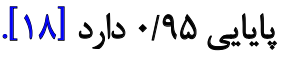

\section{هرسش نامه كيفيت زندكى}

اين يرسشنامه در بسيارى از اختلالات از جمله اماس براى

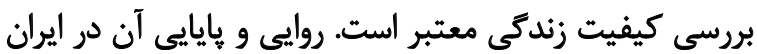

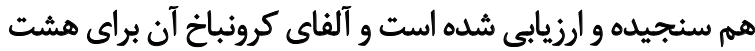

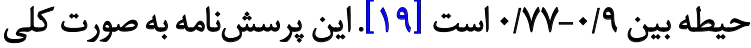

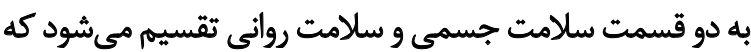

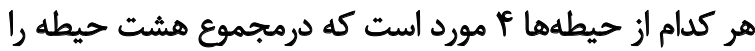

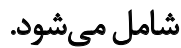

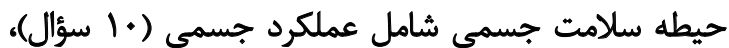

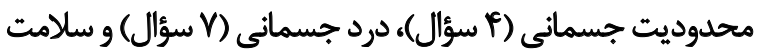

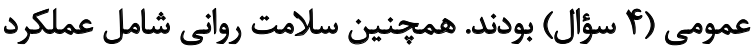

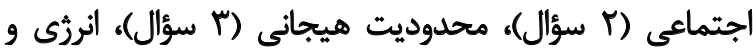

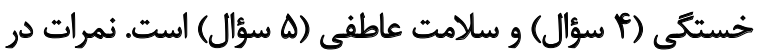

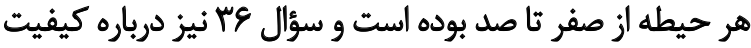
زندكى از ديدكاه خود بيمار است اصد بوده [19].]

\section{يرسش نامه تعادلى برى}

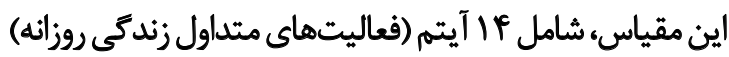

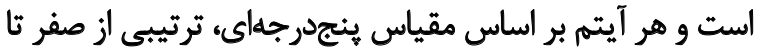

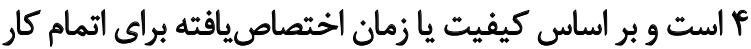

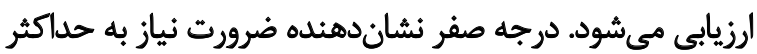

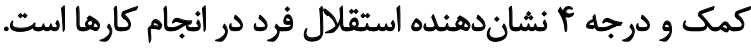

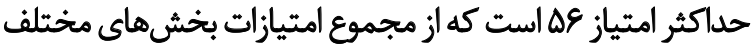

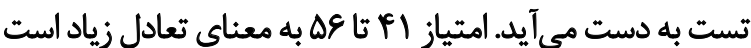

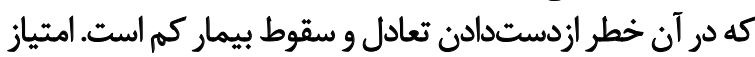

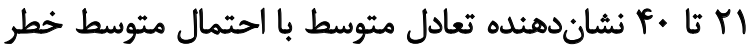

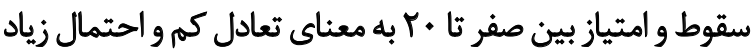

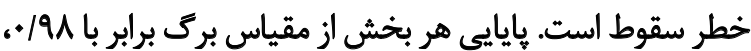

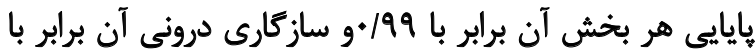

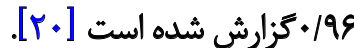

تعادل و فعاليتهاى روزمره زندگى بر كيفيت زندكى را بررسى مئى

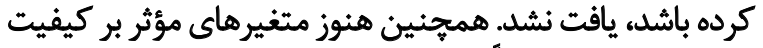

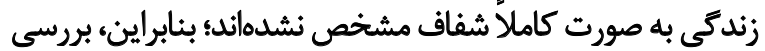

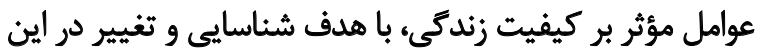

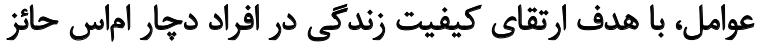

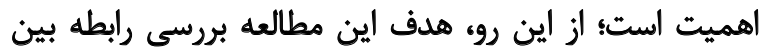

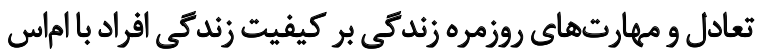

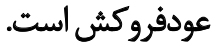
و اش بروشى

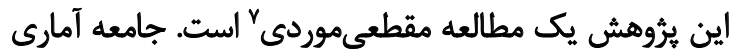

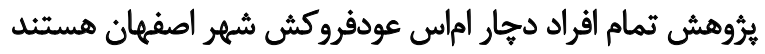

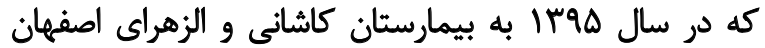

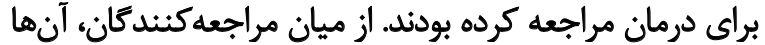

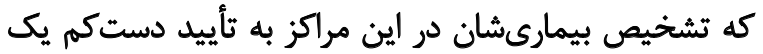

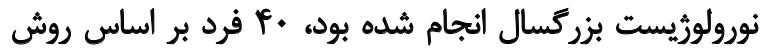

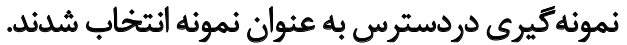

معيارهاى ورود عبارت بودند از: رضايت آزمودنىها براى شركت

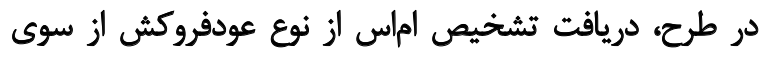

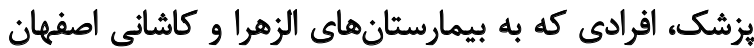

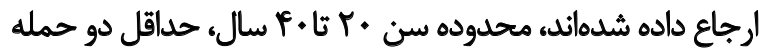

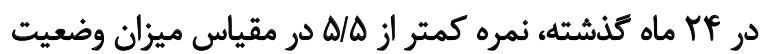

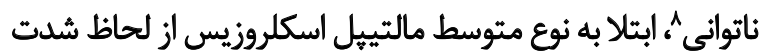

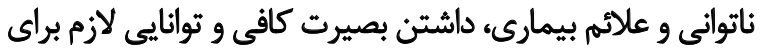

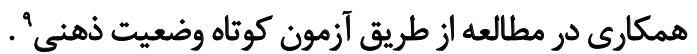
معيارهاى خروج از مطالعه عبارت بودند از: نارضايتى مراجعان

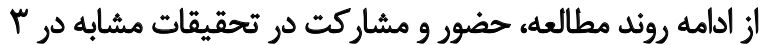

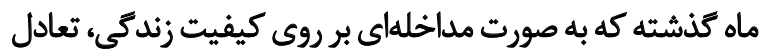

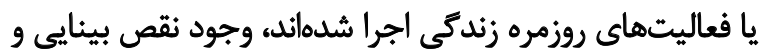

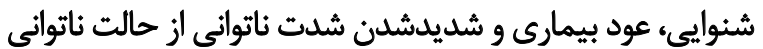

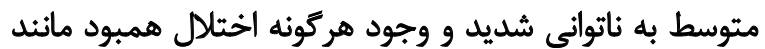

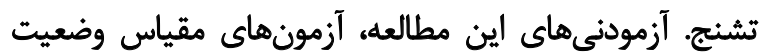

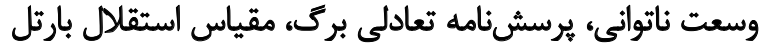
و يرسشنامه كيفيت زندكى بودند.

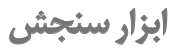
مقياس ميزان وضعيت ناتوانى

اين تست، تغييريافته آزمون مقياس ميزان وضعيت ناتوانى كورتزكه"

7. Cross Sectional

8. Expanded Disability Status Scale (EDSS)

9. Mini-Mental State Examination (MMSE)

10. Kurtzke 


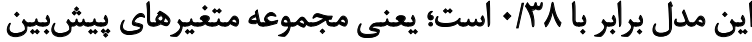

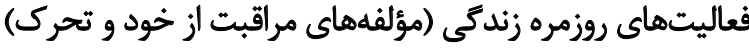

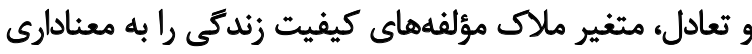

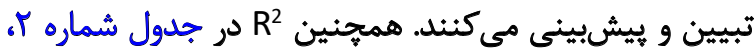

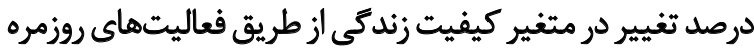

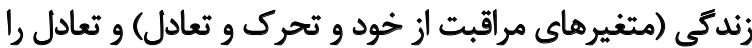

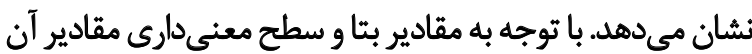

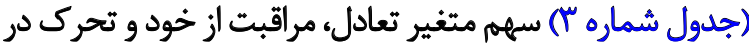

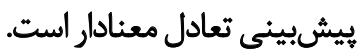

ث

هدف اين مطالعه بررسي رابطه بين تعادل و فعاليتهاي روزمره

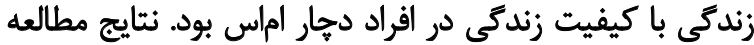

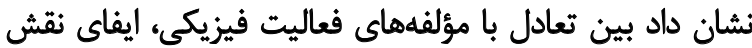

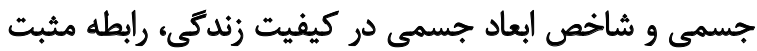

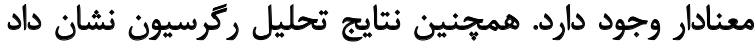
تعادل بيشبينى كننده كيفيت زندكى در بيماران دجار اماس است

نتايج اين مطالعه (رابطه بين تعادل با كيفيث زندكىى) با

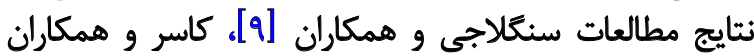

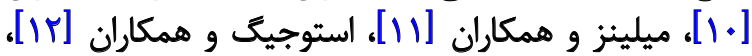

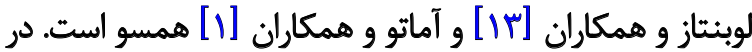

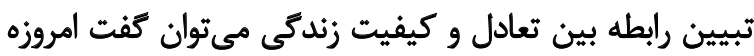

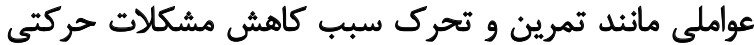

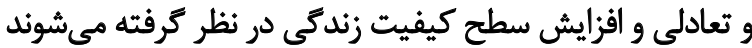

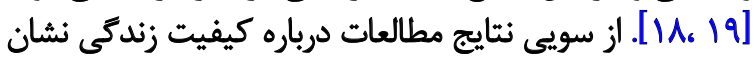

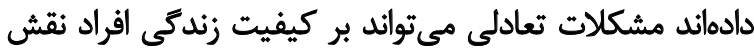

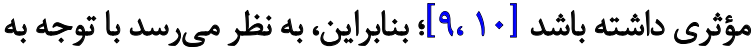

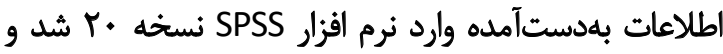

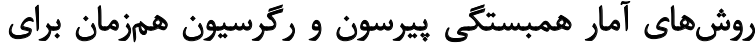
تجزيه و تحليل دادهها استفاده شد.

ياقتهها

تعداد افراد با اماس شركتكنثله ·f نفر بودند كه نتايج

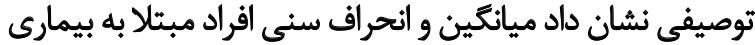

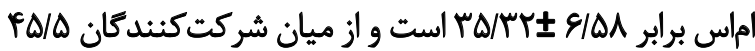

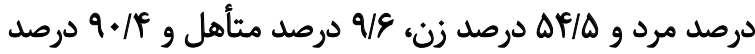
مجرد بوني.

براى بررسى رابطه بين تعادل، فعاليتهاى روزمره زندكى

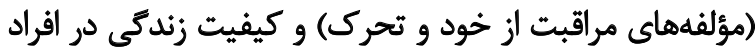

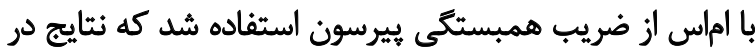

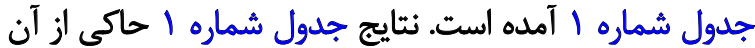

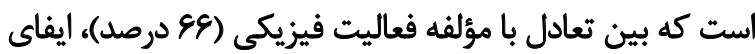

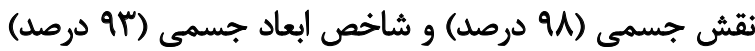

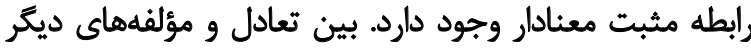

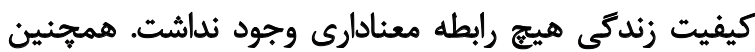

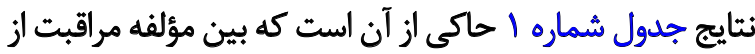

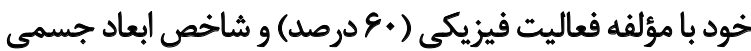

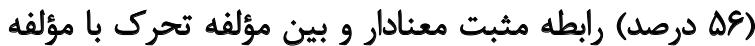

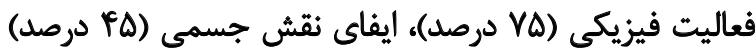

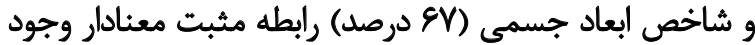

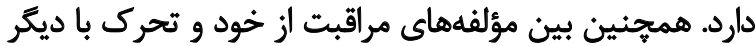

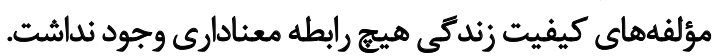
جدول شماره Y نشان مى مهد كه مقدار R تعديليافته در

جدول ا. ضريب همبستكى بين مقياس تعادلى برك، فعاليتهاى روزمره زندكى بارتل (مؤلفههاي مراقبت از خود و تحرك) و مؤلفههاى كيفيت زندكى

\begin{tabular}{|c|c|c|c|c|c|c|c|c|c|c|}
\hline شاخص ابعاد & ابعاد رواتئي & درد جسمى & سالامت & نشاط & ايفاى ثقش & اجتمأيتي & ايفاى ثقشي & از درى كلى & فيزيكيت فيتي & متغير \\
\hline Qै" & -10 & Q & $\cdot A$ & - & $M$ & $-t+$ & $u^{*}$ & $-\cdot 1$ & ge* & تعادل \\
\hline$\Delta{ }^{\circ}$ & -18 & & -.1 & $-r$. & M & -rQ & 19 & $-\cdot V$ & $80^{*}$ & مراقبت از \\
\hline$e r^{*}$ & -+1 & $-M P$ & or & $\cdot r$ & 10 & .8 & $P Q^{\circ}$ & -11 & $V D^{*}$ & فعاليت \\
\hline
\end{tabular}

جدول r. خلاصه اطلاعات جدول تحليل واريائس

\begin{tabular}{|c|c|c|c|c|c|c|c|c|}
\hline تعديلياقته R2 & $\mathbf{R}$ & $P$ & $F$ & مجئتورات & آزادجى & جمع مجذورات & شاخص آمارى & مدل \\
\hline \multirow[t]{3}{*}{.$/ R$} & IeV & $.1 . .1$ & $V(1.9)$ & FANETI & r & $\| E S / A$ & ركرسيون & \\
\hline & & & & SNHI & rv & ivala. & باقىماثده & كلى \\
\hline & & & & & f. & THOV/ME & كل & \\
\hline
\end{tabular}


جدول س. بيشبينى كيفيت زندكى از روى شاخص فعاليتهاى روزمره زندكى (مؤلفهاى مراقبت از خود و تحرك) و تعادل

\begin{tabular}{|c|c|c|c|c|}
\hline $\mathbf{P}$ & $\mathbf{T}$ & بتا & متغير & مدل \\
\hline$* 1+. r$ & $r / \Delta r$ &.$/ A T$ & تعادل & \\
\hline $.1 \cdot \pi$ & T/MEV & r/AIA & مراقبتبت الز هُود & Contant \\
\hline .1 .11 & $-T / M P$ & $-r|9|$ & فعاليت & \\
\hline
\end{tabular}

توانبخننى

نتايج اين مطالعه بيان مىكند علائم اماس تأثيرات منفى روى

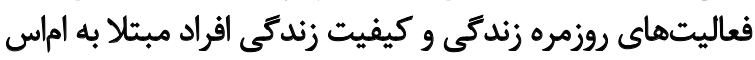

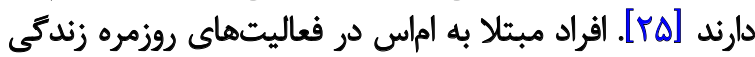

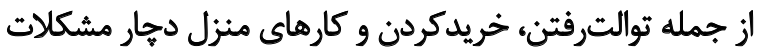

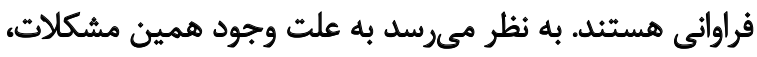

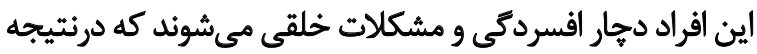

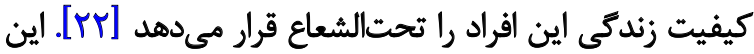

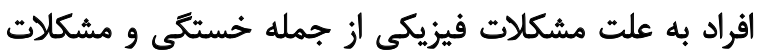

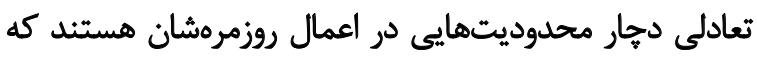

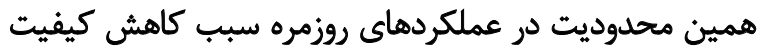

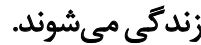

نتيجليَّيرى

با توجه به نتايج اين مطالعه كه حاكى از تأثير تعادل و استقلال

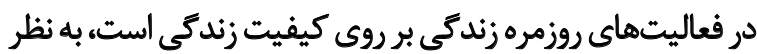

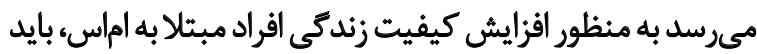

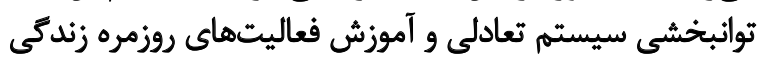
در برنامه درمانى قرار كيرد.

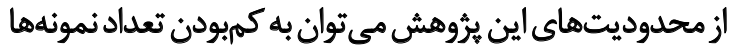

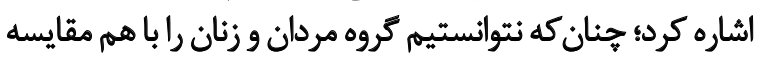

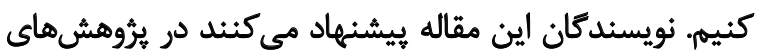

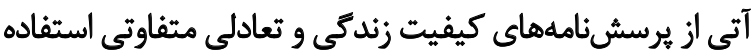
شود تانتايج تعميميذير باشد.

مالاحظات اخلاقى

\section{بيروى از اصول الخالق بثروهش}

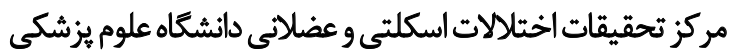

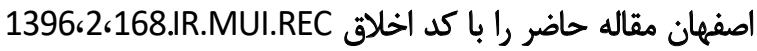

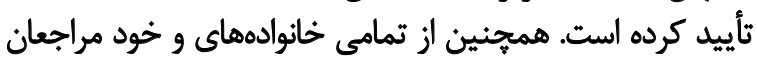

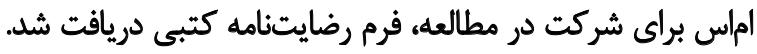

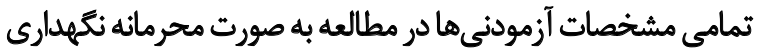

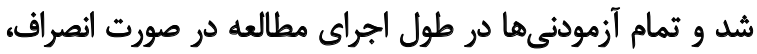
اجازه خروج داشتند.
اينكه افراد دجار اماس از يكسو به علت مشكلات تعادلى قادر به

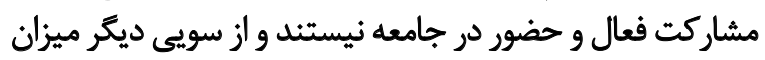

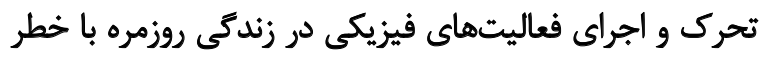

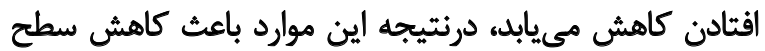

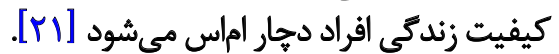

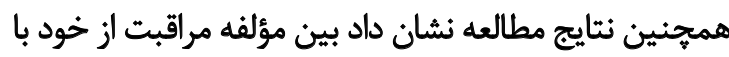

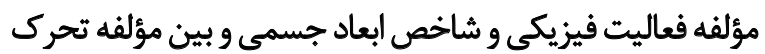

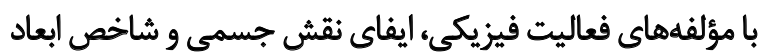

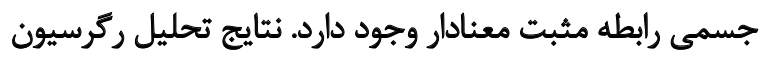

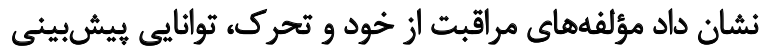

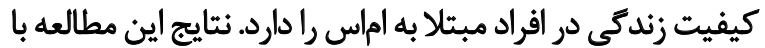

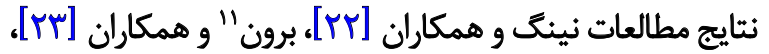

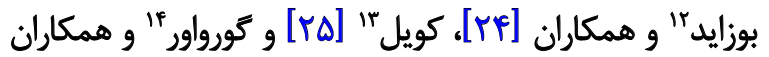

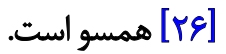

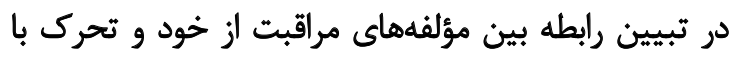

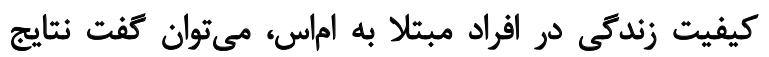

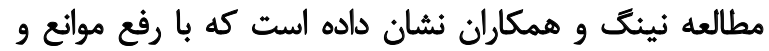

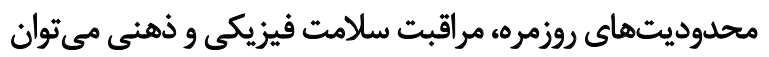

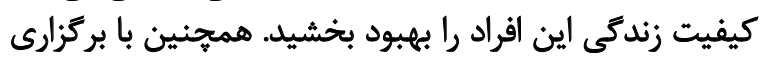

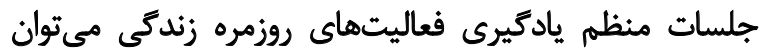

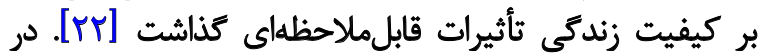

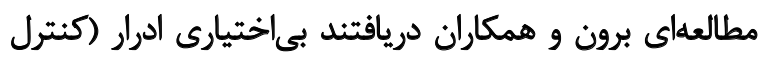

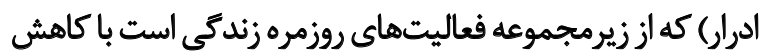
كيفيت زندكى ارتباط دارد [بrاريت.

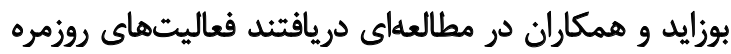

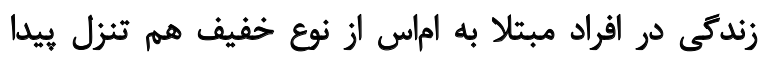

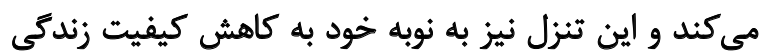

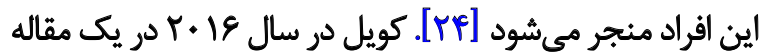

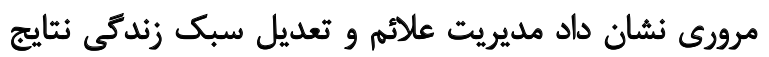

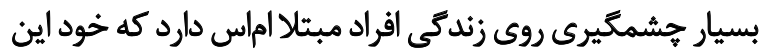

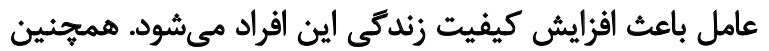

\section{Browne \\ 12. Buzaid \\ 13. Coyle}

14. Goverover 


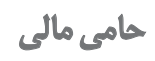

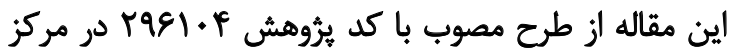

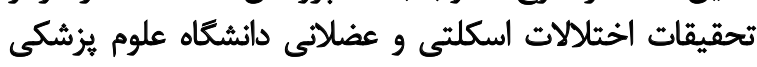
صفهان كرفته شده است.

$$
\text { مشاركت ثويسندكًان }
$$

ايدهسازى: منصور نورى و ولى شيرى؛ مفهومسازى: نازيلا اكبر فهيمى و سيد على حسينى؛ روش وشناسى: سيد على على حسئ حسينى و

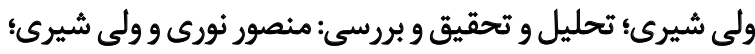

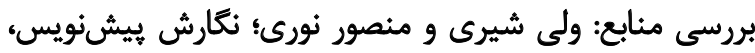

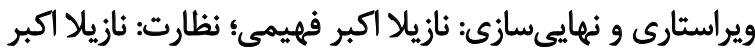

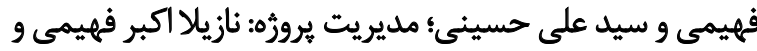
سيد على حسينى.

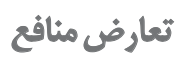

از نظر نويسندكان، اين مطالعه هيج تعارض منافعى ندارد. 


\section{References}

[1] Amato MP, Ponziani G, Rossi F, Liedl CL, Stefanile C, Rossi L. Quality of Life in Multiple Sclerosis: The impact of depression, fatigue and disability. Multiple Sclerosis Journal. 2001; 7(5):340-4. [DOI:10.1177/135245850100700511]

[2] Albrecht GL, Fitzpatrick R. Advances in medical sociology: Quality of Life in health care. Bingley: Jai Press; 1994.

[3] Kidd D, Barkhof F, McConnell R, Algra PR, Allen IV, Revesz T. Cortical lesion in Multiple Sclerosis. Journal of Neurology. 1999; 122(1):17-26. [DOI:10.1093/brain/122.1.17] [PMID]

[4] Lassmann H. The pathology of Multiple Sclerosis and its evaluartion. Philosophical Transactions of the Royal Society B: Biological Sciences. 1999; 354(1390):1635-40. [DOI:10.1098/ rstb.1999.0508] [PMID] [PMCID]

[5] Shumway Cook A, Woollacott MH. Motor control: Theory and practical application. Philadelphia: Williams \& Williams; 1995.

[6] Frzovic D, Morris ME, Vowels L. Clinical tests of standing balance: Performance of persons with Multiple Sclerosis. Archives of Physical Medicine and Rehabilitation. 2000; 81(2):215-21.

[7] American Occupational Therapy Association. Occupational therapy practice framework: Domain and process. American Journal of Occupational Therapy. 2002; 56(6):609-39. [PMID]

[8] Månsson E, Lexell J. Performance of activities of daily living in Multiple Sclerosis. Disability and Rehabilitation. 2004; 26(10):576-85.

[9] Sangelaji B, Nabavi SM, Estebsari F, Banshi MR, Rashidian H Jamshidi $E$, et al. Effect of combination exercise therapy on walking distance, postural balance, fatigue and Quality of Life in Multiple Sclerosis patients: A clinical trial study. Iranian Red Crescent Medical Journal. 2014; 16(6):e17173. [DOI:10.5812/ ircmj.17173] [PMID] [PMCID]

[10] Kasser SL, Jacobs JV, Ford M, Tourville TW. Effects of balancespecific exercises on balance, physical activity and Quality of Life in adults with Multiple Sclerosis: A pilot investigation. Disability and Rehabilitation. 2015; 37(24):2238-49. [DOI:10.3109 /09638288.2015.1019008] [PMID]

[11] Milinis K, Tennant A, Young CA, TONiC Study Group. Spasticity in Multiple Sclerosis: Associations with impairments and overall Quality of Life. Multiple Sclerosis and Related Disorders. 2016; 5:34-9. [DOI:10.1016/j.msard.2015.10.007] [PMID]

[12] Ostojic S, Stevanovic D, Jancic J. Quality of Life and its correlates in adolescent Multiple Sclerosis patients. Multiple Sclerosis and Related Disorders. 2016; 10:57-62. [DOI:10.1016/j. msard.2016.08.013] [PMID]

[13] Lobentanz IS, Asenbaum S, Vass K, Sauter C, Klösch G, Kollegger $\mathrm{H}$, et al. Factors influencing Quality of Life in Multiple Sclerosis patients: Disability, depressive mood, fatigue and sleep quality. Acta Neurologica Scandinavica. 2004; 110(1):613. [DOI:10.1111/j.1600-0404.2004.00257.x] [PMID]

[14] Rothwell PM, McDowell Z, Wong CK, Dorman PJ. Doctors and patients don't agree: Cross sectional study of patients' and doctors' perceptions and assessments of disability in Multiple Sclerosis. The BMJ. 1997; 314:1580. [DOI:10.1136/ bmj.314.7094.1580] [PMID] [PMCID]
[15] Provinciali L, Ceravolo MG, Bartolini M, Logullo F, Danni M. A multidimensional assessment of Multiple Sclerosis: Relationships between disability domains. Acta Neurologica Scandinavica. 1999; 100(3):156-62. [DOI:10.1111/j.1600-0404.1999. tb00731.x] [PMID]

[16] Heidari M, Akbarfahimi M, Salehi M, Nabavi SM. [Validity and reliability of the Persian-version of fatigue impact scale in Multiple Sclerosis patients in Iran (Persian)]. Koomesh. 2014; 15(3):295-301.

[17] Bonneville F, Moriarty DM, Li BS, Babb JS, Grossman RI, Gonen $\mathrm{O}$. Whole-brain N-Acetylaspartate concentration: Correlation with T2-weighted lesion volume and expanded disability status scale score in cases of relapsing-remitting Multiple Sclerosis. American Journal of Neuroradiology. 2002; 23(3):371-5. [PMID]

[18] Granger CV, Dewis LS, Peters NC, Sherwood CC, Barrett JE. Stroke rehabilitation: Analysis of repeated Barthel index measures. Archives of Physical Medicine and Rehabilitation. 1979; 60(1):14-7. [PMID]

[19] Montazeri A, Goshtasebi A, Vahdaninia M, Gandek B. The short Form health survey (SF-36): Translation and validation study of the Iranian version. Quality of Life Research. 2005; 14(3):875-82.

[20] Bogle Thorbahn LD, Newton RA. Use of the Berg Balance Test to predict falls in elderly persons. Physical Therapy. 1996 76(6):576-83.[DOI:10.1093/ptj/76.6.576]

[21] Turner AP, Kivlahan DR, Haselkorn JK. Exercise and Quality of Life among people with Multiple Sclerosis: Looking beyond physical functioning to mental health and participation in life. Archives of Physical Medicine and Rehabilitation. 2009; 90(3):420-8. [DOI:10.1016/j.apmr.2008.09.558] [PMID]

[22] Wu N, Minden SL, Hoaglin DC, Hadden L, Frankel D. Quality of Life in people with Multiple Sclerosis: Data from the sonya slifka longitudinal Multiple Sclerosis study. Journal of Health and Human Services Administration. 2007; 30(3):233-67. [PMID]

[23] Browne C, Salmon N, Kehoe M. Bladder dysfunction and Quality of Life for people with Multiple Sclerosis. Disability and Rehabilitation. 2015; 37(25):2350-8. [DOI:10.3109/09638288. 2015.1027007] [PMID]

[24] Buzaid A, Dodge MP, Handmacher L, Kiltz PJ. Activities of daily living: Evaluation and treatment in persons with Multiple Sclerosis. Physical Medicine and Rehabilitation Clinics of North America. 2013; 24(4):629-38. [DOI:10.1016/j. pmr.2013.06.008] [PMID]

[25] Coyle PK. Symptom management and lifestyle modifications in Multiple Sclerosis. Continuum: Lifelong Learning in Neurology. 2016; 22(3):815-36. [DOI: 10.1212/ CON.0000000000000325] [PMID]

[26] Goverover Y, Haas S, DeLuca J. Money management activities in persons with Multiple Sclerosis. Archives of Physical Medicine and Rehabilitation. 2016; 97(11):1901-7. [DOI:10.1016/j. apmr.2016.05.003] [PMID] 